\title{
ON MAPPINGS WITH DIMINISHING ORBITAL DIAMETERS
}

\author{
ZEQING LIU and SHIN MIN KANG
}

(Received 14 February 2001)

\begin{abstract}
We introduce the concepts of $*$-diminishing orbital diameters and diminishing orbital diameters for a pair $(f, g)$ of self mappings in metric spaces and prove common fixed point theorems for these mappings. The results obtained in this paper extend properly the result of Fisher (1978).
\end{abstract}

2000 Mathematics Subject Classification. 54H25.

1. Introduction. Let $f$ be a self mapping of a metric space $(X, d)$. For $x \in X$ and $A, B \subset X, \bar{A}$ denotes the closure of $A$ and let

$$
\begin{gathered}
\delta(A, B)=\sup \{d(x, y) \mid x \in A, y \in B\}, \\
\delta(A)=\delta(A, A), \quad O(x, f)=\left\{f^{n} x \mid n \in \omega\right\},
\end{gathered}
$$

where $\omega$ denotes the set of nonnegative integers. The concept of diminishing orbital diameters was introduced by Belluce and Kirk [1]. A self mapping $f$ of $(X, d)$ is said to have diminishing orbital diameters (d.o.d.) if

$$
\lim _{n \rightarrow \infty} \delta\left(O\left(f^{n} x, f\right)\right)<\delta(O(x, f)),
$$

for all $x \in X$ with $\delta(O(x, f))>0$. In compact metric spaces, Kirk [4] established the existence of fixed point for a continuous mapping with d.o.d., and Fisher [2] obtained a common fixed point theorem for a pair of contractive mappings.

Motivated by Belluce and Kirk [1] and Kirk [4], we introduce the following concepts.

DEFinITION 1.1. A pair $(f, g)$ of self mappings of a metric space $(X, d)$ is said to have $*$-diminishing orbital diameters $(*$-d.o.d.) if

$$
\lim _{n \rightarrow \infty} \delta\left(O\left(f^{n} x, f\right), O\left(g^{n} y, g\right)\right)<\delta(O(x, f), O(y, g))
$$

for all $x, y \in X$ with $\delta(O(x, f), O(y, g))>0$.

DEFINITION 1.2. A pair $(f, g)$ of self mappings of a metric space $(X, d)$ is said to have diminishing orbital diameters (d.o.d.) if

$$
\lim _{n \rightarrow \infty} \delta\left(O\left(f^{n} x, f\right), O\left(g^{n} x, g\right)\right)<\delta(O(x, f), O(x, g))
$$

for all $x \in X$ with $\delta(O(x, f), O(x, g))>0$. 
We note that $(f, g)$ has d.o.d. if $(f, g)$ has $*$-d.o.d. and that $(f, f)$ has d.o.d. if and only if $f$ has d.o.d.

The purpose of this paper is to investigate the existence of common fixed points for a pair $(f, g)$ of self mappings in compact metric spaces with either of $*$-d.o.d. and d.o.d. Our results generalize properly the result of Fisher [2]. In the sequel, $\mathbb{N}$ denotes the set of positive integers. Let $f$ be a self mapping of a metric space $(X, d)$. Define

$$
\begin{aligned}
H_{f} & =\left\{h \mid h: X \rightarrow X, h \cap_{n \in \mathbb{N}} f^{n} X \subseteq \cap_{n \in \mathbb{N}} f^{n} X\right\}, \\
L(x, f) & =\left\{w \mid \exists \text { a subsequence }\left\{f^{n_{i}} x\right\}_{i \in \mathbb{N}} \text { of }\left\{f^{n} x\right\}_{n \in \mathbb{N}}: \lim _{i \rightarrow \infty} f^{n_{i}} x=w\right\},
\end{aligned}
$$

for $x \in X$. Clearly

$$
H_{f} \supseteq C_{f}=\{h \mid h: X \longrightarrow X, h f=f h\} \supseteq\left\{f^{n} \mid n \in \omega\right\} .
$$

2. Lemmas. Leader [5] proved the following lemma.

LEMMA 2.1. Let $f$ be a continuous self mapping of a compact metric space $(X, d)$. If $A=\cap_{n \in \mathbb{N}} f^{n} X$, then $A$ is a nonempty compact subset of $X$ and $f A=A$.

LEMMA 2.2. Let $f$ and $g$ be continuous self mappings of a compact metric space $(X, d)$. If $A=\cap_{n \in \mathbb{N}} f^{n} X$ and $B=\cap_{n \in \mathbb{N}} g^{n} X$, then $\delta\left(f^{n} X, g^{n} X\right) \rightarrow \delta(A, B)$ as $n \rightarrow \infty$.

Proof. For each $n \in \mathbb{N}$, we have the following corollary.

$$
\delta\left(f^{n} X, g^{n} X\right) \geq \delta\left(f^{n+1} X, g^{n+1} X\right) \geq \delta(A, B)
$$

by $f^{n} X \supseteq f^{n+1} X \supseteq A$ and $g^{n} X \supseteq g^{n+1} X \supseteq B$. Thus $\delta\left(f^{n} X, g^{n} X\right)$ converges to some $r \geq \delta(A, B)$. Note that $f^{n} X$ and $g^{n} X$ are compact. There exist $a_{n} \in f^{n} X$ and $b_{n} \in g^{n} X$ with $\delta\left(f^{n} X, g^{n} X\right)=d\left(a_{n}, b_{n}\right)$. By the compactness of $X$, we can extract two subsequences $\left\{a_{n_{i}}\right\}_{i \in \mathbb{N}} \subset\left\{a_{n}\right\}_{n \in \mathbb{N}}$ and $\left\{b_{n_{i}}\right\}_{i \in \mathbb{N}} \subset\left\{b_{n}\right\}_{n \in \mathbb{N}}$ such that $a_{n_{i}} \rightarrow a$ and $b_{n_{i}} \rightarrow b$ as $i \rightarrow \infty$. For each $m \in \mathbb{N}$, there exists $i_{m} \in \mathbb{N}$ such that $n_{i_{m}}>m$. Consequently,

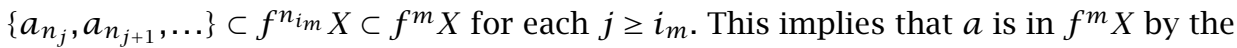
compactness of $f^{m} X$. Hence $a \in A$. Similarly we have $b \in B$. Thus

$$
\begin{aligned}
r & =\lim _{n \rightarrow \infty} \delta\left(f^{n} X, g^{n} X\right)=\lim _{i \rightarrow \infty} \delta\left(f^{n_{i}} X, g^{n_{i}} X\right) \\
& =\lim _{n \rightarrow \infty} d\left(a_{n_{i}}, b_{n_{i}}\right)=d(a, b) \\
& \leq \delta(A, B) \leq r .
\end{aligned}
$$

Therefore, $r=\delta(A, B)$. This completes the proof.

3. Fixed point theorems and examples. Our main results are as follows.

THEOREM 3.1. Let $f$ and $g$ be continuous self mapping of a compact metric space $(X, d)$. If $(f, g)$ has $*$-d.o.d., then each of $f$ and $g$ has a unique fixed point and these two points coincide. Furthermore, for each $x, y \in X$, there exist subsequences $\left\{f^{n_{i}} x\right\}_{i \in \mathbb{N}} \subset$ $\left\{f^{n} x\right\}_{n \in \mathbb{N}}$ and $\left\{g^{m_{i}} y\right\}_{i \in \mathbb{N}} \subset\left\{g^{n} y\right\}_{n \in \mathbb{N}}$ such that $\left\{f^{n_{i}} x\right\}_{i \in \mathbb{N}}$ and $\left\{g^{m_{i}} y\right\}_{i \in \mathbb{N}}$ converge to the unique fixed point of $f$. 
Proof. Let $x, y \in X$. Since $X$ is compact, $L(x, f) \neq \varnothing$. For each $t \in L(x, f)$, there exists a subsequence $\left\{f^{n_{i}} x\right\}_{i \in \mathbb{N}}$ of $\left\{f^{n} x\right\}_{n \in \mathbb{N}}$ such that $f^{n_{i}} x \rightarrow t$ as $i \rightarrow \infty$. By the continuity of $f$, we have $f^{n_{i}+1} x \rightarrow f t \in L(x, f)$. Consequently, $f L(x, f) \subseteq L(x, f)$. Note that $L(x, f)$ is closed. A Zorn's lemma argument establishes that there exists a minimal $f$-invariant nonempty closed subset $A$ of $L(x, f)$. Similarly, we can find a minimal $g$-invariant nonempty closed subset $B$ of $L(y, g)$. Note that $f$-invariance ensures that $f O(p, f) \subseteq O(p, f) \subseteq A$ for each $p \in A$. It follows from the continuity of $f$ that $f \overline{O(p, f)} \subseteq \overline{f O(p, f)} \subseteq \overline{O(p, f)}$ for $p \in A$. By the minimality of $A$, we obtain $A=\overline{O(p, f)}$. Thus $A=\overline{O\left(f^{n} p, f\right)}$ for $p \in A$ and $n \in \omega$. Similarly, we have $\overline{O(q, g)}=B=$ $\overline{O\left(g^{n} q, g\right)}$ for $q \in B$ and $n \in \omega$. We now claim that $\delta(A, B)=0$. Otherwise, $\delta(A, B)>0$. Since $(f, g)$ has $*$-d.o.d., we have

$$
\begin{aligned}
\delta(A, B) & =\delta(O(p, f), O(q, g)) \\
& >\lim _{n \rightarrow \infty} \delta\left(O\left(f^{n} p, f\right), O\left(g^{n} q, g\right)\right) \\
& =\delta(A, B),
\end{aligned}
$$

which is impossible. Hence $\delta(A, B)=0$, which implies that $A=B=$ a singleton $=\{w\}$, say. It is clear that $w$ is a common fixed point of $f$ and $g$.

If $u$ is a fixed point of $f$ and $u \neq w$. Then we obtain

$$
\begin{aligned}
d(u, w) & =\delta(O(u, f), O(w, g)) \\
& >\lim _{n \rightarrow \infty} \delta\left(O\left(f^{n} u, f\right), O\left(g^{n} w, g\right)\right) \\
& =d(u, w),
\end{aligned}
$$

which is a contradiction. Hence $w$ is a unique fixed point of $f$. Similarly, we may show that $w$ is also a unique fixed point of $g$.

Note that $w \in A \cap B \subseteq L(x, f) \cap L(y, g)$. Thus there exist subsequences $\left\{f^{n_{i}} x\right\}_{i \in \mathbb{N}} \subset$ $\left\{f^{n} x\right\}_{n \in \mathbb{N}}$ and $\left\{g^{m_{i}} y\right\}_{i \in \mathbb{N}} \subset\left\{g^{n} y\right\}_{n \in \mathbb{N}}$ such that $f^{n_{i}} x \rightarrow w$ and $g^{m_{i}} y \rightarrow w$ as $i \rightarrow \infty$. This completes the proof.

REMARK 3.2. The following example shows that if the condition that $(f, g)$ has *-d.o.d. is omitted or is replaced by the condition that $(f, g)$ has d.o.d. in Theorem 3.1, then it no longer assures the existence of a common fixed point for $f$ and $g$.

EXAMPLE 3.3. Let $X=\{1,2,3,4\}$ and $d(x, y)=|x-y|$ for $x, y \in X$. Then $(X, d)$ is a compact metric space. Define $f, g: X \rightarrow X$ as follows:

$$
f 1=f 2=g 1=2, \quad f 3=1, \quad f 4=g 3=g 4=3, \quad g 2=4 .
$$

Clearly, $f$ and $g$ are continuous. Since

$$
\lim _{n \rightarrow \infty} \delta\left(O\left(f^{n} 2, f\right), O\left(g^{n} 3, g\right)\right)=1=\delta(O(2, f), O(3, g)),
$$

it follows that $(f, g)$ has no $*$-d.o.d. Note that

$$
\lim _{n \rightarrow \infty} \delta\left(O\left(f^{n} x, f\right), O\left(g^{n} x, g\right)\right)=\delta(\{2\},\{3\})=1<2 \leq \delta(O(x, f), O(x, g)),
$$

for all $x \in X$. That is, $(f, g)$ has d.o.d. But $f$ and $g$ do not have a common fixed point. 
As a particular case of Theorem 3.1 we have the following corollary.

COROLlaRY 3.4. Let $f$ be a continuous self mapping of a compact metric space $(X, d)$. If $(f, f)$ has $*$-d.o.d., then $f$ has a unique fixed point. Furthermore, for each $x \in X$ there exists some subsequence of $\left\{f^{n} x\right\}_{n \in \mathbb{N}}$ converges to the unique fixed point of $f$.

QUESTION 3.5. Let $f$ satisfy all the conditions of Corollary 3.4 and $C=\cap_{n \in \mathbb{N}} f^{n} X$. Does $C$ contain exactly one point?

THEOREM 3.6. Let $f$ and $g$ be continuous self mappings of a compact metric space $(X, d)$ satisfying for some $r, s \in \mathbb{N}$,

$$
d\left(f^{r} x, g^{s} y\right)<\delta\left(\cup_{h \in H_{f}} h O(x, f), \cup_{t \in H_{g}} t O(y, g)\right),
$$

for all $x, y \in X$ for which the right-hand side of (3.6) is positive. Then

(i) each of $f$ and $g$ has a uniformly contractive fixed point and these two points coincide.

(ii) $H_{f}$ and $H_{g}$ have a unique common fixed point.

(iii) $(f, g)$ has both $*$-d.o.d. and d.o.d.

Proof. Let $A=\cap_{n \in \mathbb{N}} f^{n} X$ and $B=\cap_{n \in \mathbb{N}} g^{n} X$. We assert that $\delta(A, B)=0$. If not, then $\delta(A, B)>0$. By Lemma 2.1, $f A=A \neq \varnothing, g B=B \neq \varnothing, A$ and $B$ are compact. Thus there exist $a, u \in A$ and $b, v \in B$ such that $\delta(A, B)=d(a, b), a=f^{r} u$ and $b=g^{s} v$. Clearly $a \in \cup_{h \in H_{f}} h O(u, f)$ and $b \in \cup_{t \in H_{g}} t O(v, g)$. Using (3.6) we have

$$
\begin{aligned}
\delta(A, B) & =d\left(f^{r} u, g^{s} v\right) \\
& <\delta\left(\cup_{h \in H_{f}} h O(u, f), \cup_{t \in H_{g}} t O(v, g)\right) \\
& \leq \delta(A, B),
\end{aligned}
$$

which is absurd. Hence $\delta(A, B)=0$. This implies that $A=B=$ a singleton. Thus (i) follows from Theorem 1 of Leader [5].

We now show that (ii) holds. Let $A=B=\{w\}$. It is easy to see that $H_{f}$ and $H_{g}$ have a common fixed point $w$. Suppose that $v$ is a common fixed point of $H_{f}$ and $H_{g}$ and $v \neq w$. From (3.6) we get

$$
\begin{aligned}
d(v, w) & =d\left(f^{r} v, g^{s} w\right) \\
& <\delta\left(\cup_{h \in H_{f}} h O(v, g), \cup_{t \in H_{g}} t O(w, g)\right) \\
& =d(v, w),
\end{aligned}
$$

which is a contradiction. Hence $H_{f}$ and $H_{g}$ have a unique common fixed point.

We next show that (iii) holds. Assume that $x, y$ are in $X$ with $\delta(O(x, f), O(y, g))>0$. Since $O\left(f^{n} x, f\right) \subset f^{n} X$ and $O\left(g^{n} y, g\right) \subset g^{n} X$, we have by Lemma 2.2

$$
\begin{aligned}
\delta\left(O\left(f^{n} x, f\right), O\left(g^{n} y, g\right)\right) & \leq \delta\left(f^{n} X, g^{n} X\right) \\
& \longrightarrow \delta(A, B) \\
& =0
\end{aligned}
$$


as $n \rightarrow \infty$. It follows that

$$
\lim _{n \rightarrow \infty} \delta\left(O\left(f^{n} x, f\right), O\left(g^{n} y, g\right)\right)=0<\delta(O(x, f), O(y, g)) .
$$

Hence $(f, g)$ has $*$-d.o.d. This implies that $(f, g)$ has also d.o.d. This completes the proof.

As in the proof of Theorem 3.6, we obtain the following theorem.

THEOREM 3.7. Let $f$ be a continuous self mapping of a compact metric space $(X, d)$ satisfying for some $r, s \in \mathbb{N}$,

$$
d\left(f^{r} x, f^{s} y\right)<\delta\left(\cup_{h \in H_{f}} h(O(x, f) \cup O(y, f))\right),
$$

for all $x, y \in X$ for which the right-hand side of (3.11) is positive. Then

(i) $f$ has a uniformly contractive fixed point $w$. In fact, $w=h w$ for $h \in H_{f}$.

(ii) $(f, f)$ has both $*$-d.o.d. and d.o.d.

REMARK 3.8. Corollary 4.3 of Jungck [3] is a special case of Theorem 3.7(i).

From Theorem 3.6 we have the following corollary.

COROLLARY 3.9. Let $f$ and $g$ be continuous self mappings of a compact metric space $(X, d)$ satisfying

$$
d\left(f^{2} x, g^{2} y\right)<\delta\left(\cup_{h \in H_{f}} h O(x, f), \cup_{t \in H_{g}} t O(y, g)\right),
$$

for all $x, y \in X$ for which the right-hand side of (3.12) is positive. Then (i), (ii), and (iii) of Theorem 3.6 hold.

Fisher [2] obtained the following result.

THEOREM 3.10. Suppose that $f$ and $g$ are continuous self mappings of a compact metric space $(X, d)$ satisfying either

$$
\begin{aligned}
d\left(f^{2} x, g^{2} y\right)< & \max \{d(x, g y), d(y, f x), d(x, y)\} \\
& \text { if } \max \{d(x, g y), d(y, f x), d(x, y)\} \neq 0,
\end{aligned}
$$

or

$$
d\left(f^{2} x, g^{2} y\right)=0 \quad \text { if } \max \{d(x, g y), d(y, f x), d(x, y)\}=0
$$

for all $x, y \in X$. Then $f$ and $g$ have a unique common fixed point.

REMARK 3.11. Note that $\max \{d(x, g y), d(y, f x), d(x, y)\}=0$ implies $x=y=$ $f x=g y=f^{2} x=g^{2} y$. Hence condition (3.14) of Theorem 3.10 can be omitted. It is easy to see that (3.13) implies (3.12). The following example reveals that Corollary 3.9 extend properly Theorem 3.10. 
EXAMPLE 3.12. Let $X=\{1,2,3,4,5\}$ with the usual metric. Define $f, g: X \rightarrow X$ by

$$
\begin{gathered}
f 3=g 3=2, \quad g 4=3, \quad f 2=g 1=4, \\
f 1=f 4=f 5=g 2=g 5=5 .
\end{gathered}
$$

Then $f$ and $g$ are continuous self mappings of a compact metric space $(X, d)$. It is easy to see that

$$
\delta\left(\cup_{h \in H_{f}} h O(x, f), \cup_{t \in H_{g}} t O(y, g)\right)=0
$$

if and only if $(x, y)=(5,5)$. It is now a simple matter to show that

$$
d\left(f^{2} x, g^{2} y\right) \leq 3<4=\delta\left(\cup_{h \in H_{f}} h O(x, f), \cup_{t \in H_{g}} t O(y, g)\right),
$$

for $(x, y) \neq(5,5)$. Thus the assumptions of Corollary 3.9 are satisfied. But Theorem 3.10 is not applicable since

$$
d\left(f^{2} f, g^{2} g\right)=1=\max \{d(3, g 3), d(3, f 3), d(3,3)\},
$$

that is, $f$ and $g$ do not satisfy (3.13) for $x=y=3$.

ACKNOWLEDGEMENTS. The authors thank the referee for providing useful comments and suggestions on improving the original version of this paper, and the second author was supported by Korea Research Foundation Grant (KRF-99-005-D00003).

\section{REFERENCES}

[1] L. P. Belluce and W. A. Kirk, Fixed-point theorems for certain classes of nonexpansive mappings, Proc. Amer. Math. Soc. 20 (1969), 141-146. MR 38\#1663. Zbl 165.16801.

[2] B. Fisher, Common fixed point mappings on complete and compact metric spaces, Math. Japon. 22 (1978), no. 5, 565-569. MR 80a:54080. Zbl 379.54019.

[3] G. Jungck, Common fixed points for commuting and compatible maps on compacta, Proc. Amer. Math. Soc. 103 (1988), no. 3, 977-983. MR 89h:54030. Zbl 661.54043.

[4] W. A. Kirk, On mappings with diminishing orbital diameters, J. London Math. Soc. 44 (1969), 107-111. MR 38\#1664. Zbl 162.54902.

[5] S. Leader, Uniformly contractive fixed points in compact metric spaces, Proc. Amer. Math. Soc. 86 (1982), no. 1, 153-158. MR 83j:54041. Zbl 507.54040.

ZeQing LiU: DePartment of MATHEMatics, LiaOning Normal UniVersity, Dalian, LIAONING 116029, CHINA

E-mail address: zeqing 7 iu@sina.com.cn

Shin Min Kang: Department of Mathematics, Gyeongsang National University, CHINJU 660-701, KOREA

E-mail address: smkang@nongae.gsnu.ac.kr 


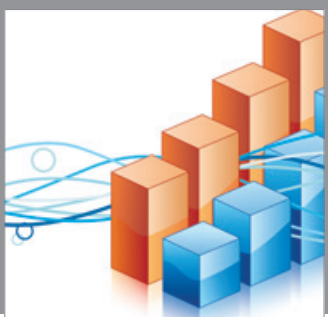

Advances in

Operations Research

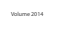

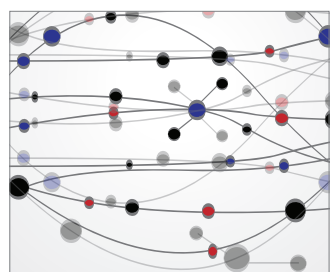

\section{The Scientific} World Journal
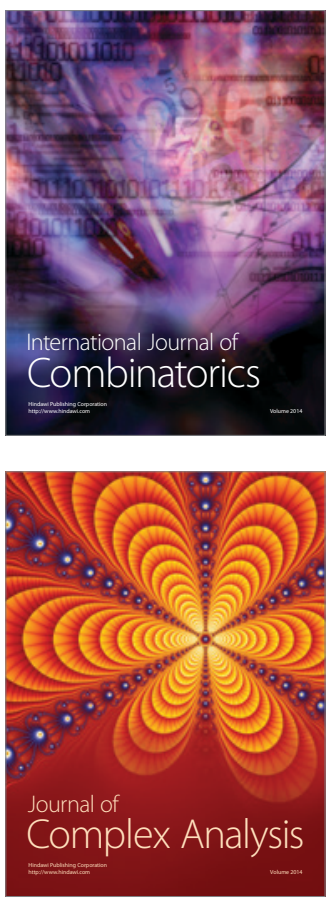

International Journal of

Mathematics and

Mathematical

Sciences
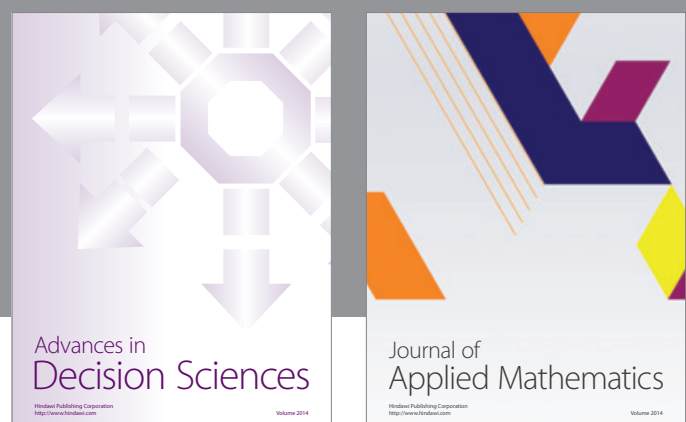

Journal of

Applied Mathematics
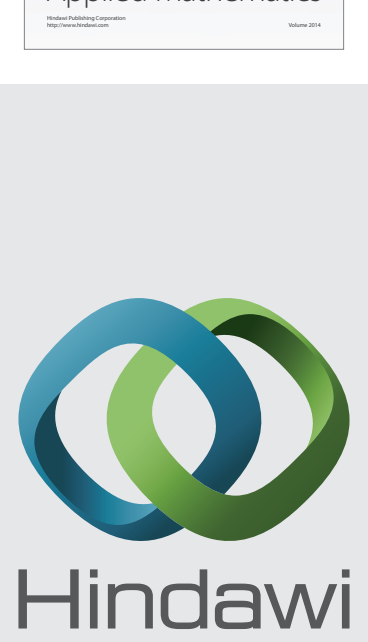

Submit your manuscripts at http://www.hindawi.com
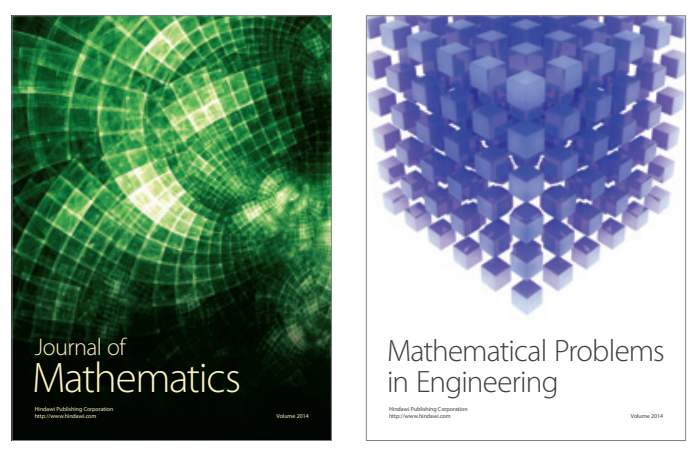

Mathematical Problems in Engineering
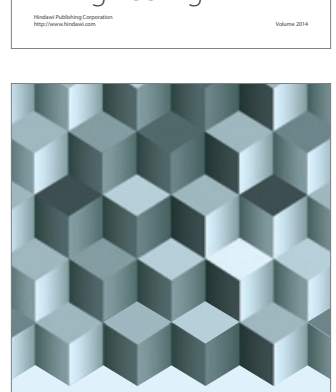

Journal of

Function Spaces
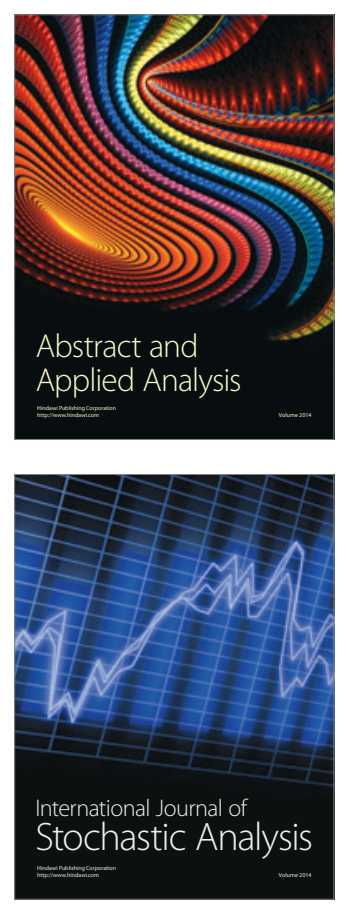

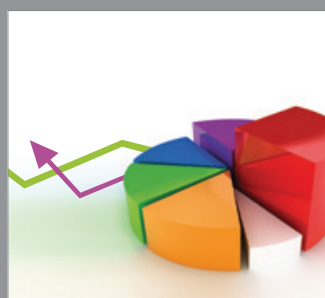

ournal of

Probability and Statistics

Promensencen
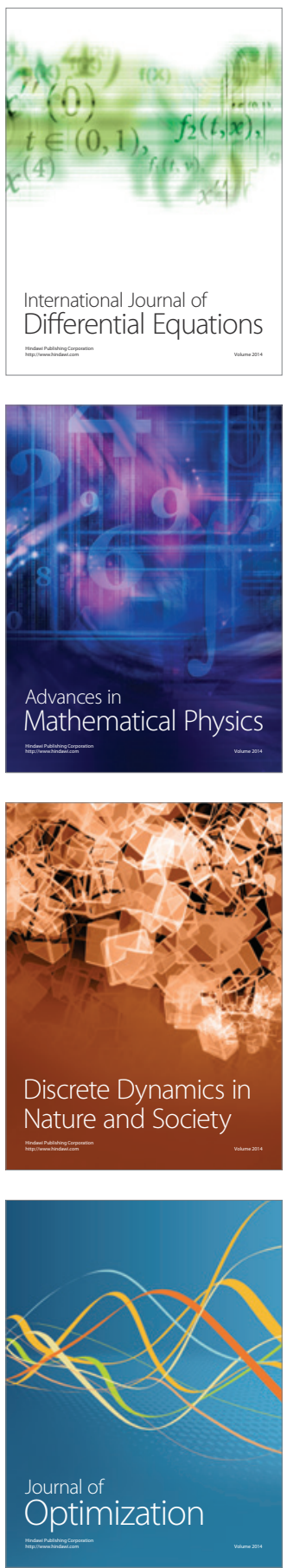